\title{
Comparison of the Effect of Nitroglycerin, Magnesium Sulphate and Dexmedetomidine as Hypotensive Agents in Lumbar Spine Surgery
}

Osama Helal Ahmed, Tawfik Mohamed Nour-Eldin, Waheed Mohamed Ali, Marwa Ali Abd El Zaher*

Department of Anesthesiology and Intensive Care, Faculty of Medicine, Al-Azhar University, Assuit, Egypt

*Corresponding Author: Marwa Ali Abd El zaher, Phone No.: (+2) 01065856550, E-mail: amarwa23@yahoo.com

\begin{abstract}
Background: in orthopedic procedures more blood is lost from raw bone and muscle surface than from identifiable blood vessels. Moderate hypotensive anesthesia was found to significantly decrease the average blood loss by nearly $40 \%$, reduce the need for transfusion by $45 \%$ and shorten the average operating time by nearly $10 \%$.

Objective: The aim of the current study was to compare magnesium sulphate and dexmedetomidine with nitroglycerin as regard hypotensive effect as primary outcome, volume of blood loss, blood substitution and pattern of recovery as secondary outcome during lumbar spine surgery. Patients and Methods: This prospective, controlled, comperative, randomized, double blind study included a total of ninty patients aged 21-50 years of both sex, ASA I-II scheduled for elective lumber spine surgery, attending at Department of Orthopedic, AL-Azher university Hospital in Assuit as single center study. Patients have received either dexmedetomidine, magnesium sulfate or nitroglycerine.

Results: There were highly significant difference $(\mathrm{P}<0.000)$ with duration of surgery between different study groups with duration of surgery shortest in dexmedetomidine group followed by magnesium sulfate group and then nitroglycerine group. There were highly significant differences between different study groups with fluid maintenance with higher volume in nitroglycerine group then magnesium sulfate group and then dexmedetomidine group. There were highly significant differences $(\mathrm{P}<0.000)$ with systolic blood pressure between study groups at $\mathrm{A} 1$ and hypotensive agent discontinuation with lowest systolic blood pressure in dexmedetomidine group followed by magnesium sulfate group and then nitroglycerine group.
\end{abstract}

Conclusion: nitroglycerine, magnesium sulfate and dexmedetomidine could induce hypotension, but dexmedetomidine showed more favorable hemodynamic profile as regard blood pressure and heart rate.

Keywords: Nitroglycerin, Magnesium Sulphate, Dexmedetomidine, Hypotensive agents, Lumbar spine surgery.

\section{INTRODUCTION}

In orthopedic procedures more blood is lost from raw bone and muscle surfaces than from identifiable blood vessels. A radioactive isotope study of blood loss in major orthopedic procedures showed that estimates of blood losses were, on average, $50 \%$ of the true measured loss ${ }^{(1)}$. Laminectomy and spinal fusion are associated with increased blood loss due to bleeding from the extensive epidural venous plexuses and poses possibility of nerve injury root level if not visualized properly. Nerve roots are at jeopardy during laminectomy and decrease hemorrhage adds to safety of surgery in this area ${ }^{(2)}$.

Moderate hypotensive anaesthesia was found to significantly decrease the average blood loss by nearly $40 \%$, reduce the need for transfusion by nearly $45 \%$ and shorten the average operating time by nearly $10 \%{ }^{(2)}$.

Deliberate hypotension is defined as the intentional reduction of the systemic perfusion pressure. Deliberate hypotension is defined as reduction in systolic blood pressure (SBP) to $80-90 \mathrm{~mm} \mathrm{Hg} \mathrm{(30 \%} \mathrm{decrease} \mathrm{in}$ the SBP from the baseline pressure) or a decrease in the mean arterial pressure (MAP) to $50-65 \mathrm{~mm} \mathrm{Hg}$ in normotensive patients ${ }^{(3)}$.

Many anesthetic agents and vasoactive drugs are used frequently to produce controlled hypotension, including inhalational anesthetics, direct-acting vasodilators, autonomic ganglion blockers, $\beta$-adrenergic blockers, and calcium channel blockers ${ }^{(4)}$. It was in 1985 M Guggiari et al., used nitroglycerine (NTG) for the first time to produce induced hypotension in aneurismal brain surgery and proved that it can be used as a sole agent for hypotension ${ }^{(5)}$.
The decrease in arterial pressure is achieved by vasodilatory effect of NTG on arterial and venous bed resulting finally in decreased venous and right heart filling and so decreased cardiac output.Nitroglycerine causes either no change or slight tachycardia during continuous infusion as slight increase in heart rate is reflex phenomenon, baroreceptor response secondary to hypotension produced ${ }^{(\boldsymbol{(})}$.

I.V. magnesium sulphate may be a good agent for deliberate hypotension because magnesium intervenes in the activation of membrane $\mathrm{Ca}$ ATPase and $\mathrm{Na}-\mathrm{K}$ ATPase involved in transmembrane ion exchanges during depolarization and repolarization phases, and thus act as a stabilizer of cell membrane and intracytoplasmic organelles ${ }^{(7)}$.The antagonist effect of magnesium at $\mathrm{N}$ methyl-D-aspartate (NMDA) receptors has led to studies of its adjuvant effect in perioperative analgesia (8). Dexmedetomidine (DEX) is a potent highly selective $\alpha_{2}$ adrenergic agonist, possessing a differential specificity for the $\alpha_{2}: \alpha_{1}$ receptors ${ }^{(9)}$. DEX has sedative, analgesic, anesthetic sparing effect, and sympatholytic properties (10). The central and peripheral sympatholytic action of DEX is mediated by $\alpha_{2}$ adrenergic receptors ${ }^{(11)}$ and is manifested by dose-dependent decrease in arterial blood pressure, heart rate, cardiac output and norepinephrine release ${ }^{(\mathbf{1 0})}$.

The aim of the current study was to compare magnesium sulphate and dexmedetomidine with nitroglycerin as regard hypotensive effect as primary outcome, volume of blood loss, blood substitution and pattern of recovery as secondary outcome during lumbar spine surgery. 


\section{PATIENTS AND METHODS}

This prospective, controlled, comperative, randomized, double blind study included a total of ninty patients aged 21-50 years of both sex, ASA I-II scheduled for elective lumber spine surgery, attending at Department of Orthopedic, AL-Azher university Hospital in Assuit. Written informed consent from all the subjects were obtained. This study was conducted between January 2017 to February 2018.

Ethical approval:

\section{Approval of the Hospital Ethics Committee was} obtained.

\section{Exclusion criteria:}

- ASA grade III, IV, uncontrolled diabetes mellitus, pulmonary disease, uncontrolled hypertension, ischemic heart disease, gastro-esophageal reflux disease, cerebral ischemia and renal impairment.

- History of difficult airway management.

- Body mass index $>40$, history of neuromuscular disease, pregnancy.

- Known allergy to all study drugs.

- Prior treatment with calcium channel blockers, opioids, anticoagulants and patients receiving magnesium supplementation or drugs known to have a significant interaction with NMDAs.

- Duration of surgery $>150 \mathrm{~min}$

\section{Grouping:}

According to computer program and use of opaque sealed envelope, the included subjects were randomally allocated into three equal groups, 30 subjects each; Group N (nitroglycerin, control group), Group M (magnisum sulfate group) and group $\mathrm{D}$ (dexmedetomidine group).

Preparation: Patients have received either dexmedetomidine, magnesium sulfate or nitroglycerine.

Group N (controls) received saline IV starting 10 minutes before the beginning of surgery and nitroglycerine infusion started after induction.

Group D received dexmedetomidine IVstarting 10 minutes before the beginning of surgery and continued during the operation.

Group $\mathrm{M}$ received $\mathrm{MgSO}_{4}$ IV starting 10 minutes before the beginning of surgery and continued during the operation. Target blood pressure lowering 30\%. More than that we stop the study drug.

\section{Premedication:}

All patients were premedicated with IV midazolam (Dormicum, Roche,Switzerland) $0.02 \mathrm{mg} / \mathrm{kg}$ after insertion of $20 \mathrm{G}$ peripheral IV catheter.

Group N (controls) (Glyceryl Trinitrate ,Hameln pharma plus gmbh, Germany) received $25 \mathrm{ml} 0.9 \%$ saline infused over 10 minutes then received $3 \mu \mathrm{g} / \mathrm{kg} / \mathrm{min}$ and was titrated to achieve and maintain the desired hypotension. Group D received loading dose of $1 \mu \mathrm{g}$ dexmedetomidine (Precedex, sigma, united states) diluted in $25 \mathrm{ml} 0.9 \%$ saline infused over 10 minutes followed by contiuous IV infusion of $0.2 \mu \mathrm{g} / \mathrm{kg} / \mathrm{h}$.
Group M (Magnasium sulphate, Egypation international pharmaceutical, Egypt) the infusion started before induction at loading dose of $25 \mathrm{mg} / \mathrm{kg}$ diluted in 25 $\mathrm{ml} 0.9 \%$ saline for 10 minutes and then was sustained throughout operation at maintainance dose of $15 \mathrm{mg} / \mathrm{kg} /$ $\mathrm{h}$ intravenously.

\section{Anesthesia technique:}

All patients received the same anesthetic technique using fentanyl $2 \mu \mathrm{g} / \mathrm{kg}$ IV and propfol $1-2$ $\mathrm{mg} / \mathrm{kg} \mathrm{IV}$ (till lose of eye lash reflex). The intubation was facilitated by the use of rocuronium $0.6 \mathrm{mg} / \mathrm{kg}$ IV.

\section{Maintenance:}

Group D received continuous IV infusion of $0.2 \mu \mathrm{g} / \mathrm{kg} / \mathrm{h}$. Group M infusion was sustained throughout operation at maintainance dose of $15 \mathrm{mg} / \mathrm{kg} / \mathrm{h}$ intravenously.

In control group $(\mathrm{N})$ the induction started with 3 $\mu \mathrm{g} / \mathrm{kg} / \mathrm{min}$ and was titrated to achieve and maintain desired hypotension. Anesthesia was maintained with $0.5-1 \%$ end-tidal isoflurane in $100 \%$ oxygen. Adequate muscle relaxation was maintained with incremental bolus doses of $0.1-0.2 \mathrm{mg} / \mathrm{kg}$ IV rocuronium when indicated by peripheral nerve stimulation. Controlled ventilation was adjusted to maintain normocapnia $(30-35 \mathrm{mmHG}) j$

Fluid therapy included maintenance plus deficit fluids that was replaced over the first 3 to 4 hours of the procedure and third space losses which was replaced by $6 \mathrm{ml} / \mathrm{kg} / \mathrm{h}$.

\section{Monitoring:}

Continuous monitoring of invasive blood pressure, non-invasive blood pressure in PACU, HR, Spo2, ETCO2, ECG, urine output and sudden changes in plans.

Parameters of study: Data collected to compare between the three groups (DEX, $\mathrm{Mgso}_{4}$ and nitroglycerin):

- Demographic data

- Blood loss estimated by Fromme score and blood transfusion

- Pulse rate at time of induction, at start of hypotensive agent, 15, 30, 45, 60 minutes, at hypotensive agent discontinuation and after extubation.

- Blood pressure (systolic, diastolic and mean) at time of induction, at start of hypotensive agent, 15, $30,45,60$ minutes, at hypotensive agent discontinuation and after extubation.

- $\mathrm{O}_{2}$ saturation, arterial blood gases and UOP at time of induction, at start of hypotensive agent, 15, 30, 45, 60 minutes, at hypotensive agent discontinuation and after extubation.

- Time required to restore MAP to baseline after discontinuation of the study drug

- At the end of surgery groups was compared with reference to blood loss by fromme score

- Modified aldert score

- Time to $1^{\text {st }}$ analgesic rescue in PACU

- Blood pressure, pulse rate, $\mathrm{O}_{2}$ saturation, $\mathrm{ETCO}_{2}$, UOP in PACU. 


\section{Statistical analysis}

- SPSS program (statistical package for social science) will be used for data entry and analysis.

- Mean and standard deviation will be used for expression of quantative data, percent will be used for expression of qualitative data.

- Anova and chi-square will be used to assess difference between the three groups.

\section{RESULTS}

Table (1) shows Demographic data in study groups. There were non significance differences $(\mathrm{P}>0.05)$ between age, sex, weight, height and BMI.

Table (1): Demographic data in study groups.

\begin{tabular}{|l|c|c|c|c|}
\hline \multicolumn{1}{|c|}{ Item } & $\mathbf{N}$ & $\mathbf{M}$ & $\mathbf{D}$ & P-value \\
\hline 1-Age (years) & $42.50 \pm 6.94$ & $44.86 \pm 13.96$ & $48.43 \pm 12.20$ & $\mathrm{P}=0.204 \mathrm{n} . \mathrm{s}$ \\
2-Sex: & & & & \\
Male & $15(50.0 \%)$ & $22(73.3 \%)$ & $13(43.3 \%)$ & $\mathrm{P}=0.448 \mathrm{n} . \mathrm{s}$ \\
Female & $15(50.0 \%)$ & $8(20.0 \%)$ & $17(56.7 \%)$ & \\
3- Weight (kg) & $83.00 \pm 6.51$ & $85.33 \pm 6.28$ & $78.67 \pm 7.97$ & $\mathrm{P}=0.264 \mathrm{n} . \mathrm{s}$ \\
4-Height $(\mathbf{c m})$ & $160.00 \pm 7.34$ & $175.00 \pm 4.54$ & $167.40 \pm 5.01$ & $\mathrm{P}=0.437 \mathrm{n} . \mathrm{s}$ \\
5-BMI $\left(\mathbf{k g} / \mathbf{m}^{2}\right)$ & $26.84 \pm 4.46$ & $27.87 \pm 1.96$ & $28.02 \pm 2.04$ & $\mathrm{P}=0.214 \mathrm{n} . \mathrm{s}$ \\
\hline
\end{tabular}

* Significant

** Moderatly significant

*** Highly significant

Table (2) shows clinical data in study groups. There were highly significance differences $(\mathrm{P}<0.000)$ between different study groups with each of duration of surgery. Also there were moderate significance difference $(\mathrm{P}<0.001)$ with fluid maintenance.

Table (2): Clinical data in study groups.

\begin{tabular}{|l|c|c|c|c|}
\hline \multicolumn{1}{|c|}{ Item } & $\mathbf{N}$ & $\mathbf{M}$ & $\mathbf{D}$ & P-value \\
\hline 1- Duration of surgery (hr) & $2.25 \pm 0.13$ & $1.89 \pm 0.26$ & $1.33 \pm 0.45$ & $\mathrm{P}<0.000 * * *$ \\
2- Fluid maintenance (ml) & & & & \\
& $188.00 \pm 23.01$ & $123.00 \pm 6.51$ & $122.67 \pm 14.95$ & $\mathrm{P}<0.001 * *$ \\
\hline
\end{tabular}

Table (3) shows systolic blood pressure in study groups. There were highly significance difference $(\mathrm{P}<0.000)$ between study groups at A1 \& A5. There were significance difference between study groups $(\mathrm{P}<0.05)$ at $\mathrm{A} 15 \& \mathrm{~A} 30$. But there were non significance difference $(\mathrm{P}>0.05)$ between study groups at other times.

In group $\mathrm{N}$ when comparing with baseline. There were highly significance difference $(\mathrm{P}<0.000)$ with $\mathrm{A} 60$, there were moderate significance difference $(\mathrm{P}<0.001)$ at A45, also there were significance difference $(\mathrm{P}<0.05)$ with $\mathrm{A} 15$ \& $\mathrm{A} 30$.

In group $\mathrm{M}$ when comparing with baseline. There were moderate significance difference $(\mathrm{P}<0.001)$ at $\mathrm{A} 45$, also there were significance difference $(\mathrm{P}<0.05)$ with A5, A15, A30 \& A45.

In group $\mathrm{D}$ when comparing with baseline. There were significance difference $(\mathrm{P}<0.05)$ with A45 \& A60.
When comparing between group $\mathrm{N} \&$ group $\mathrm{M}$, there were moderate significance difference $(\mathrm{P}<0.001)$ between study groups at A15 \& Hypotensive agent discontinuation, also there were significance difference between study groups $(\mathrm{P}<0.05)$ at $\mathrm{A} 30 \& \mathrm{~A} 45$. But there were non significance differences $(\mathrm{P}>0.05)$ between study groups at other times.

When comparing between group $\mathrm{N} \&$ group $\mathrm{D}$ There were moderate significance there were non significance difference $(\mathrm{P}>0.05)$ between study groups at different times.

When comparing between group $\mathrm{M} \&$ group $\mathrm{D}$ There were moderate significance difference $(\mathrm{P}<0.001)$ between study groups at A15 \& Hypotensive agent discontinuation, also there were significance difference between study groups $(\mathrm{P}<0.05)$ at $\mathrm{A} 15, \mathrm{~A} 30$ \& $\mathrm{A} 45$. But there were non significance difference $(\mathrm{P}>0.05)$ between study groups at other times. 
Table (3): Systolic blood pressure in $\mathrm{mmHg}$ in study groups.

\begin{tabular}{|c|c|c|c|c|}
\hline Item & $\mathbf{N}$ & $\mathbf{M}$ & $\mathbf{D}$ & P-value \\
\hline & $118.86 \pm 18.22$ & $110.00 \pm 5.73$ & $105.03 \pm 4.08$ & $\mathrm{P}<0.005^{* *}$ \\
4-A30 & $110.67 \pm 14.76$ & $98.80 \pm 8.75^{*}$ & $94.50 \pm 7.04$ & $\mathrm{P}<0.004^{* *}$ \\
5-A45 & $105.80 \pm 11.00^{*}$ & $97.90 \pm 6.02^{*}$ & $93.10 \pm 8.61$ & $\mathrm{P}<0.04^{*}$ \\
6-A60 & $101.93 \pm 10.74^{*}$ & $98.50 \pm 8.30^{*}$ & $94.16 \pm 8.75$ & $\mathrm{P}<0.04^{*}$ \\
7-Hypotensive agent & $97.60 \pm 13.81^{* *}$ & $89.60 \pm 11.88^{* *}$ & $88.56 \pm 10.23^{*}$ & $\mathrm{P}=0.385 \mathrm{n} . \mathrm{s}$ \\
discontinuation & $95.20 \pm 13.77^{* * *}$ & $93.90 \pm 6.45^{*}$ & $90.20 \pm 9.44^{*}$ & $\mathrm{P}=0.254 \mathrm{n} . \mathrm{s}$ \\
8-After extubation & $118.40 \pm 11.92$ & $111.00 \pm 9.76$ & $99.63 \pm 11.67$ & $\mathrm{P}=0.424 \mathrm{n} . \mathrm{s}$ \\
& & & & \\
& $129.73 \pm 7.71$ & $125.00 \pm 13.15$ & $124.43 \pm 17.31$ & $\mathrm{P}=0.245 \mathrm{n} . \mathrm{s}$ \\
\hline
\end{tabular}

Intragroup:*significant,

**moderetly significant,

***highly significant

\begin{tabular}{|l|c|c|c|}
\hline \multicolumn{1}{|c|}{ Item } & P1\&P2 & P1\&P3 & P2\&P3 \\
\hline 1-A1 & $\mathrm{P}=0.265 \mathrm{n} . \mathrm{s}$ & $\mathrm{P}=0.084 \mathrm{n} . \mathrm{s}$ & $\mathrm{P}<0.000^{* * *}$ \\
2-A5 & $\mathrm{P}=0.168 \mathrm{n} . \mathrm{s}$ & $\mathrm{P}=0.966 \mathrm{n} . \mathrm{s}$ & $\mathrm{P}<0.04^{*}$ \\
3-A15 & $\mathrm{P}<0.004^{* *}$ & $\mathrm{P}=0.211 \mathrm{n} . \mathrm{s}$ & $\mathrm{P}<0.01^{*}$ \\
4-A30 & $\mathrm{P}<0.02^{*}$ & $\mathrm{P}=0.565 \mathrm{n} . \mathrm{s}$ & $\mathrm{P}<0.04^{*}$ \\
5-A45 & $\mathrm{P}<0.04 *$ & $\mathrm{P}=0.138 \mathrm{n} . \mathrm{s}$ & $\mathrm{P}=0.719 \mathrm{n} . \mathrm{s}$ \\
6-A60 & $\mathrm{P}=0.195 \mathrm{n} . \mathrm{s}$ & $\mathrm{P}=0.914 \mathrm{n} . \mathrm{s}$ & $\mathrm{P}=0.082 \mathrm{n} . \mathrm{s}$ \\
7-Hypotensive agent discontinuation & $\mathrm{p}<0.001^{*}$ & $\mathrm{P}=0.832 \mathrm{n} . \mathrm{s}$ & $\mathrm{P}<0.000^{* * *}$ \\
8-After extubation & $\mathrm{p}=0.131 \mathrm{n} . \mathrm{s}$ & $\mathrm{P}=0.095 \mathrm{n} . \mathrm{s}$ & $\mathrm{P}=0.887 \mathrm{n} . \mathrm{s}$ \\
\hline
\end{tabular}

Table (4) shows diastolic blood pressure in study groups. There were moderate significance difference $(\mathrm{P}<0.001)$ between study groups at A30. But there were non significance difference $(\mathrm{P}>0.05)$ between study groups at other times. In group $\mathrm{N}$ when comparing with baseline. There were significance difference $(\mathrm{P}<0.05)$ with A45 \& A60.

In group $\mathrm{M}$ when comparing with baseline. There were significance difference $(\mathrm{P}<0.05)$ with $\mathrm{A} 15$, A30, A45 \& A60. In group D when comparing with baseline. There were moderate significance difference $(\mathrm{P}<0.001)$ with A30.
When comparing between group N\& groupM There were non significance difference $(\mathrm{P}>0.05)$ between study groups at different times.

When comparing between group N\& groupD There were moderate significance there were significance difference $(\mathrm{P}<0.05)$ between study groups at A30.

When comparing between group $\mathrm{M} \&$ groupD. There were highly significance difference $(\mathrm{P}<0.000)$ at A30. But there were non significance difference $(\mathrm{P}>0.05)$ between study groups at other times. 
Table (4): Diastolic blood pressure in $\mathrm{mmHg}$ in study groups.

\begin{tabular}{|l|c|c|c|c|}
\hline Item & $\mathbf{N}$ & $\mathbf{M}$ & $\mathbf{D}$ & P-value \\
\hline 1-A1 & $71.80 \pm 9.59$ & $73.33 \pm 12.31$ & $76.23 \pm 11.14$ & $\mathrm{P}=0.294 \mathrm{n} . \mathrm{s}$ \\
2-A5 & $69.40 \pm 9.01$ & $66.67 \pm 13.55$ & $64.96 \pm 8.71^{*}$ & $\mathrm{P}=0.463 \mathrm{n} . \mathrm{s}$ \\
3-A15 & $67.80 \pm 11.39$ & $64.80 \pm 12.14^{*}$ & $61.96 \pm 13.25^{*}$ & $\mathrm{P}=0.942 \mathrm{n} . \mathrm{s}$ \\
4-A30 & $68.10 \pm 6.29$ & $62.93 \pm 10.62^{*}$ & $59.50 \pm 10.30^{* *}$ & $\mathrm{P}<0.002 * *$ \\
5-A45 & $65.50 \pm 12.95^{*}$ & $63.00 \pm 11.86^{*}$ & $59.16 \pm 11.53^{* *}$ & $\mathrm{P}=0.403 \mathrm{n} . \mathrm{s}$ \\
6-A60 & $62.40 \pm 10.90^{*}$ & $64.33 \pm 13.23^{*}$ & $60.23 \pm 13.14^{* *}$ & $\mathrm{P}=0.448 \mathrm{n} . \mathrm{s}$ \\
7- Hypotensive agent & $70.50 \pm 10.59$ & $70.73 \pm 14.42$ & $70.16 \pm 13.31$ & $\mathrm{P}=0.985 \mathrm{n} . \mathrm{s}$ \\
discontinuation & & & & \\
8-After extubation & $78.40 \pm 8.37$ & $81.60 \pm 8.23$ & $80.67 \pm 14.69$ & $\mathrm{P}=0.505 \mathrm{n} . \mathrm{s}$ \\
\hline
\end{tabular}

Intragroup:*significant

**moderetly significant

$* * *$ Highly significant

\begin{tabular}{|c|c|c|c|}
\hline Item & P1\&P2 & P1\&P3 & P2\&P3 \\
\hline & $\mathrm{P}=0.343 \mathrm{n} . \mathrm{s}$ & $\mathrm{P}=0.593 \mathrm{n} . \mathrm{s}$ & $\mathrm{P}=0.104 \mathrm{n} . \mathrm{s}$ \\
& $\mathrm{P}=0.566 \mathrm{n} . \mathrm{s}$ & $\mathrm{P}=0.562 \mathrm{n} . \mathrm{s}$ & $\mathrm{P}=0.139 \mathrm{n} . \mathrm{s}$ \\
& $\mathrm{P}=0.960 \mathrm{n} . \mathrm{s}$ & $\mathrm{P}=0.743 \mathrm{n} . \mathrm{s}$ & $\mathrm{P}=0.716 \mathrm{n} . \mathrm{s}$ \\
& $\mathrm{P}=0.209 \mathrm{n} . \mathrm{s}$ & $\mathrm{P}<0.02^{*}$ & $\mathrm{P}<0.000 * * *$ \\
& $\mathrm{P}=0.210 \mathrm{n} . \mathrm{s}$ & $\mathrm{P}=0.280 \mathrm{n} . \mathrm{s}$ & $\mathrm{P}=0.917 \mathrm{n} . \mathrm{s}$ \\
& $\mathrm{P}=0.233 \mathrm{n} . \mathrm{s}$ & $\mathrm{P}=0.539 \mathrm{n} . \mathrm{s}$ & $\mathrm{P}=0.490 \mathrm{n} . \mathrm{s}$ \\
otensive agent discontinuation & $\mathrm{P}=0.875 \mathrm{n} . \mathrm{s}$ & $\mathrm{P}=0.943 \mathrm{n} . \mathrm{s}$ & $\mathrm{P}=0.915 \mathrm{n} . \mathrm{s}$ \\
& & & \\
r extubation & $\mathrm{P}=0.763 \mathrm{n} . \mathrm{s}$ & $\mathrm{P}=0.141 \mathrm{n} . \mathrm{s}$ & $\mathrm{P}=0.466 \mathrm{n} . \mathrm{s}$ \\
\hline
\end{tabular}

Table (5) shows time required to restore the MAP to baseline after discontinuation of the study drug \& time to first analgesic rescue in PACU. There were highly significance $(\mathrm{P}<0.000)$ with time required to restore the MAP to baseline after discontinuation of the study drug \& time to first analgesic rescue in PACU.

Table (5): Time required to restore the MAP to baseline after discontinuation of the study drug \& time to first analgesic rescue in PACU.

\begin{tabular}{|c|c|c|c|c|}
\hline Item & $\mathbf{N}$ & $\mathbf{M}$ & $\mathbf{D}$ & P-value \\
\hline $\begin{array}{l}\text { 1- Time required to restore } \\
\text { the MAP to baseline (min) } \\
\text { 2- Time to first analgesic } \\
\text { rescue in PACU (min) }\end{array}$ & $11.90 \pm 2.59$ & $15.67 \pm 2.53$ & $17.70 \pm 2.36$ & $\mathrm{P}<0.000 * * *$ \\
\hline
\end{tabular}

Table (6) shows Heart in study groups. There were moderate significance difference $(\mathrm{P}<0.001)$ between study groups at after extubation. Also there were significance difference $(\mathrm{P}<0.05)$ At time A5 \& A15. But there were non significance difference $(\mathrm{P}>0.05)$ between study groups at other times.

In group $\mathrm{N}$ when comparing with baseline. There were significance difference $(\mathrm{P}>0.05)$ in different times.

In group $\mathrm{M}$ when comparing with baseline. There were significance difference $(\mathrm{P}<0.05)$ in after extubation and non-significance difference $(\mathrm{P}>0.05)$ at different times.

In group $\mathrm{D}$ when comparing with baseline. There were moderate significance difference
$(\mathrm{P}<0.001)$ with after extubation. Also, there were significance difference $(\mathrm{P}<0.05)$ with A5 \& A15.

When comparing between group N\& groupM

There were non significance difference $(\mathrm{P}>0.05)$ between study groups at different times.

When comparing between group $\mathrm{N} \&$ groupD There were non significance difference $(P>0.05)$ between study groups at different times.

When comparing between group $\mathrm{M} \&$ groupD. There were highly significance difference $(\mathrm{P}<0.000)$ at After extubation. But there were non significance differences $(\mathrm{P}>0.05)$ between study groups at other times. 
Table (6): Heart rate in beat/min in study groups.

\begin{tabular}{|l|c|c|c|c|}
\hline \multicolumn{1}{|c|}{ Item } & $\mathbf{N}$ & $\mathbf{M}$ & $\mathbf{D}$ & P-value \\
\hline 1-A1 & $101.30 \pm 11.32$ & $77.46 \pm 12.65$ & $60.20 \pm 12.53$ & $\mathrm{P}=0.084 \mathrm{n} . \mathrm{s}$ \\
2-A5 & $105.70 \pm 10.18$ & $82.26 \pm 16.42$ & $62.06 \pm 12.62$ & $\mathrm{P}<0.04^{*}$ \\
3-A15 & $102.40 \pm 11.69$ & $76.80 \pm 12.91$ & $62.83 \pm 13.48$ & $\mathrm{P}<0.017^{*}$ \\
4-A30 & $104.40 \pm 10.92$ & $78.33 \pm 15.65$ & $68.36 \pm 9.44$ & $\mathrm{P}=0.930 \mathrm{n} . \mathrm{s}$ \\
5-A45 & $103.60 \pm 12.90$ & $76.26 \pm 16.33$ & $69.53 \pm 9.97$ & $\mathrm{P}=0.509 \mathrm{n} . \mathrm{s}$ \\
6-A60 & $102.30 \pm 10.57$ & $76.00 \pm 14.85$ & $71.90 \pm 11.25$ & $\mathrm{P}=0.609 \mathrm{n} . \mathrm{s}$ \\
7- Hypotensive agent discontinuation & $100.50 \pm 11.84$ & $79.40 \pm 11.60$ & $78.60 \pm 15.81^{* *}$ & $\mathrm{P}=0.827 \mathrm{n} . \mathrm{s}$ \\
8-After extubation & $98.70 \pm 10.37$ & $96.13 \pm 13.16^{*}$ & $90.16 \pm 10.48^{* *}$ & $\mathrm{P}<0.003^{* *}$ \\
\hline
\end{tabular}

Intragroup:*significant

$* *$ moderetly significant

$* * *$ highly significant

\begin{tabular}{|l|l|l|l|}
\hline \multicolumn{1}{|c|}{ Item } & P1\&P2 & P1\&P3 & P2\&P3 \\
\hline 1-A1 & $\mathrm{P}=0.319 \mathrm{n} . \mathrm{s}$ & $\mathrm{P}=0.221 \mathrm{n} . \mathrm{s}$ & $\mathrm{P}<0.02^{*}$ \\
2-A5 & $\mathrm{P}=0.062 \mathrm{n} . \mathrm{s}$ & $\mathrm{P}=0.903 \mathrm{n} . \mathrm{s}$ & $\mathrm{P}<0.01^{*}$ \\
3-A15 & $\mathrm{P}=0.249 \mathrm{n} . \mathrm{s}$ & $\mathrm{P}=0.084 \mathrm{n} . \mathrm{s}$ & $\mathrm{P}<0.005^{* *}$ \\
4-A30 & $\mathrm{P}=0.992 \mathrm{n} . \mathrm{s}$ & $\mathrm{P}=0.760 \mathrm{n} . \mathrm{s}$ & $\mathrm{P}=0.697 \mathrm{n} . \mathrm{s}$ \\
5-A45 & $\mathrm{P}=0.718 \mathrm{n} . \mathrm{s}$ & $\mathrm{P}=0.486 \mathrm{n} . \mathrm{s}$ & $\mathrm{P}=0.192 \mathrm{n} . \mathrm{s}$ \\
6-A60 & $\mathrm{P}=0.398 \mathrm{n} . \mathrm{s}$ & $\mathrm{P}=0.928 \mathrm{n} . \mathrm{s}$ & $\mathrm{P}=0.360 \mathrm{n} . \mathrm{s}$ \\
7-Hypotensive agent discontinuation & $\mathrm{P}=0.739 \mathrm{n} . \mathrm{s}$ & $\mathrm{P}=0.491 \mathrm{n} . \mathrm{s}$ & $\mathrm{P}=804 \mathrm{n} . \mathrm{s}$ \\
8-After extubation & $\mathrm{P}=0.074 \mathrm{n} . \mathrm{s}$ & $\mathrm{P}=0.141 \mathrm{n} . \mathrm{s}$ & $\mathrm{P}<0.000^{* * *}$ \\
\hline
\end{tabular}

Table (7) shows systolic blood pressure in study groups. "PACU". There were highly significance difference $(\mathrm{P}<0.000)$ between groups at different time.

In group $\mathrm{N}$ there were significance difference $(\mathrm{P}<0.05)$ at time $30 \& 35$ mins. As regard there were moderate significance difference $(\mathrm{P}<0.001)$ at times $40-60 \mathrm{mins}$.

In group $M$ there were significance difference $(\mathrm{P}<0.05)$ at time $55 \& 60$ mins.

In group $\mathrm{D}$ there were significance difference $(\mathrm{P}<0.05)$ at time $25-60$ mins.

When comparing group N\&M there were highly significance difference $(\mathrm{P}<0.000)$ at time $10-60 \mathrm{mins}$.

When comparing $\mathrm{N} \& \mathrm{D}$ there were significance difference $(\mathrm{P}<0.05)$ at time 55-60mins.

When comparing $M \& D$ there were significance difference $(\mathrm{P}<0.05)$ at time 25-60 mins.

Table (7): Systolic blood pressure in $\mathrm{mmHg}$ in study groups. "PACU"

\begin{tabular}{|l|c|c|c|c|}
\hline Item & $\mathbf{N}$ & $\mathbf{M}$ & $\mathbf{D}$ & $\mathbf{P}$-value \\
\hline 1-5mins & $117.70 \pm 9.25$ & $108.67 \pm 6.33$ & $109.06 \pm 7.39$ & $\mathrm{P}<0.000^{* * *}$ \\
2-10mins & $116.90 \pm 5.23$ & $106.73 \pm 5.48$ & $102.40 \pm 2.01$ & $\mathrm{P}<0.000^{* * *}$ \\
3-15mins & $115.30 \pm 2.98$ & $106.13 \pm 6.15$ & $102.13 \pm 2.88$ & $\mathrm{P}<0.000^{* * *}$ \\
4-20mins & $114.00 \pm 2.31$ & $106.00 \pm 5.09$ & $103.06 \pm 2.42$ & $\mathrm{P}<0.000^{* * *}$ \\
5-25mins & $112.00 \pm 2.22$ & $106.20 \pm 6.35$ & $101.23 \pm 2.56^{*}$ & $\mathrm{P}<0.000^{* * *}$ \\
6-30mins & $110.80 \pm 2.56^{*}$ & $106.60 \pm 5.83$ & $101.36 \pm 3.11^{*}$ & $\mathrm{P}<0.000^{* * *}$ \\
7-35mins & $109.30 \pm 1.70^{*}$ & $106.67 \pm 5.67$ & $102.86 \pm 3.02^{*}$ & $\mathrm{P}<0.000^{* * *}$ \\
8-40mins & $108.90 \pm 2.69^{* *}$ & $106.93 \pm 5.33$ & $100.50 \pm 5.73^{*}$ & $\mathrm{P}<0.000^{* * *}$ \\
9-45mins & $107.70 \pm 2.65^{* *}$ & $105.86 \pm 6.76$ & $99.53 \pm 4.08^{*}$ & $\mathrm{P}<0.000^{* * *}$ \\
10-50mins & $107.10 \pm 1.15^{* *}$ & $103.73 \pm 5.15$ & $100.76 \pm 3.72^{*}$ & $\mathrm{P}<0.000^{* * *}$ \\
11-55mins & $106.90 \pm 0.93^{* *}$ & $102.13 \pm 4.91^{*}$ & $100.86 \pm 5.25^{*}$ & $\mathrm{P}<0.000^{* * *}$ \\
12-60mins & $105.90 \pm 2.20^{* *}$ & $101.13 \pm 4.19^{*}$ & $100.36 \pm 5.38^{*}$ & $\mathrm{P}<0.000^{* * *}$ \\
\hline
\end{tabular}

Intragroup:*significant

**moderetly significant

***highly significant 
Table (7): Systolic blood pressure in mmHg in study groups. "PACU"

\begin{tabular}{|l|c|c|c|}
\hline Item & $\mathbf{P 1 \& P 2}$ & $\mathbf{P 1 \& P 3}$ & $\mathbf{P 2 \& P 3}$ \\
\hline 1-5mins & $\mathrm{P}=0.823 \mathrm{n} . \mathrm{s}$ & $\mathrm{P}<0.001^{* *}$ & $\mathrm{P}<0.01^{*}$ \\
2-10mins & $\mathrm{P}<0.000^{* * *}$ & $\mathrm{P}<0.001^{* *}$ & $\mathrm{P}<0.001^{* *}$ \\
3-15mins & $\mathrm{P}<0.002^{* *}$ & $\mathrm{P}<0.001^{* *}$ & $\mathrm{P}<0.01^{*}$ \\
4-20mins & $\mathrm{P}<0.006^{* *}$ & $\mathrm{P}<0.01^{*}$ & $\mathrm{P}<0.01^{*}$ \\
5-25mins & $\mathrm{P}<0.000^{* * *}$ & $\mathrm{P}<0.000^{* * *}$ & $\mathrm{P}<0.01^{*}$ \\
6-30mins & $\mathrm{P}=0.583 \mathrm{n} . \mathrm{s}$ & $\mathrm{P}<0.001^{* *}$ \\
7-35mins & $\mathrm{P}<0.000^{* * *}$ & $\mathrm{P}=0.526 \mathrm{n} . \mathrm{s}$ & $\mathrm{P}<0.001^{* *}$ \\
8-40mins & $\mathrm{P}=0.261 \mathrm{n} . \mathrm{s}$ & $\mathrm{P}<0.01^{*}$ \\
9-45mins & $\mathrm{P}<0.000^{* * *}$ & $\mathrm{P}=0.336 \mathrm{n} . \mathrm{s}$ & $\mathrm{P}<0.01^{*}$ \\
10-50mins & $\mathrm{P}<0.000^{* * *}$ & $\mathrm{P}=0.475 \mathrm{n} . \mathrm{s}$ & $\mathrm{P}<0.02^{*}$ \\
11-55mins & $\mathrm{P}<0.000^{* * *}$ & $\mathrm{P}=0.584 \mathrm{n} . \mathrm{s}$ & $\mathrm{P}<0.01^{*}$ \\
12-60mins & $\mathrm{P}<0.000^{* * *}$ & $\mathrm{P}=0.586 \mathrm{n} . \mathrm{s}$ & $\mathrm{P}<0.02^{*}$ \\
\hline
\end{tabular}

Table (8) shows Diastolic blood pressure in study groups. "PACU". There were highly significance difference $(\mathrm{P}<0.000)$ between groups at different times.

When comparing between group N\& groupM. There were highly significance difference $(\mathrm{P}<0.000)$ at different times.

When comparing between group $\mathrm{N} \&$ groupD. There were highly significance difference $(\mathrm{P}<0.000)$ at
$25 \mathrm{mns}$., also there were moderate significance difference $(\mathrm{P}<0.001)$ at times 5- $15 \mathrm{mns}$. As regard there were significance difference $(\mathrm{P}<0.05)$ at time 20 , there were non significance difference $(\mathrm{P}>0.05)$ at other times.

When comparing group $\mathrm{M} \&$ groupD there were moderate significance difference $(\mathrm{P}<0.001)$ at times 10 , $30 \& 35 \mathrm{mns}$. Also, there were significance difference $(\mathrm{P}<0.05)$ at other times.

Table (8): Diastolic blood pressure in $\mathrm{mmHg}$ in study groups. "PACU"

\begin{tabular}{|l|c|c|c|c|}
\hline \multicolumn{1}{|c|}{ Item } & $\mathbf{N}$ & $\mathbf{M}$ & $\mathbf{D}$ & P-value \\
\hline 1-5mins & $73.90 \pm 8.46$ & $70.93 \pm 7.50$ & $62.50 \pm 12.46$ & $\mathrm{P}<0.000^{* * *}$ \\
2-10mins & $70.70 \pm 4.77$ & $68.67 \pm 8.60$ & $58.43 \pm 5.98$ & $\mathrm{P}<0.000^{* * *}$ \\
3-15mins & $65.90 \pm 6.09$ & $67.67 \pm 6.67$ & $58.10 \pm 7.31$ & $\mathrm{P}<0.000^{* * *}$ \\
4-20mins & $66.50 \pm 4.07$ & $58.67 \pm 7.06$ & $57.03 \pm 6.02$ & $\mathrm{P}<0.000^{* * *}$ \\
5-25mins & $63.90 \pm 5.42$ & $57.00 \pm 8.26$ & $56.80 \pm 4.05$ & $\mathrm{P}<0.000^{* * *}$ \\
6-30mins & $62.50 \pm 4.10$ & $58.33 \pm 5.77$ & $56.13 \pm 3.73$ & $\mathrm{P}<0.000^{* * *}$ \\
7-35mins & $65.50 \pm 5.30$ & $59.33 \pm 7.15$ & $55.36 \pm 3.96$ & $\mathrm{P}<0.000^{* * *}$ \\
8-40mins & $64.00 \pm 5.93$ & $56.00 \pm 7.92$ & $56.23 \pm 5.56$ & $\mathrm{P}<0.000^{* * *}$ \\
9-45mins & $65.00 \pm 7.54$ & $58.66 \pm 7.97$ & $56.40 \pm 3.59$ & $\mathrm{P}<0.000^{* * *}$ \\
10-50mins & $63.50 \pm 4.57$ & $58.00 \pm 5.18$ & $57.36 \pm 3.89$ & $\mathrm{P}<0.000^{* * *}$ \\
11-55mins & $64.50 \pm 4.79$ & $56.67 \pm 6.60$ & $57.76 \pm 3.75$ & $\mathrm{P}<0.000^{* * *}$ \\
12-60mins & $65.00 \pm 4.54$ & $58.00 \pm 5.18$ & $58.10 \pm 3.04$ & $\mathrm{P}<0.000^{* * *}$ \\
\hline
\end{tabular}

Intragroup:*significant

**moderetly significant

***highly significant

\begin{tabular}{|l|c|c|c|}
\hline \multicolumn{1}{|c|}{ Item } & P1\&P2 & P1\&P3 & P2\&P3 \\
\hline 1-5mins & $\mathrm{P}=0.823 \mathrm{n} . \mathrm{s}$ & $\mathrm{P}<0.001^{* *}$ & $\mathrm{P}<0.01^{*}$ \\
2-10mins & $\mathrm{P}<0.000^{* * *}$ & $\mathrm{P}<0.001^{* *}$ & $\mathrm{P}<0.001^{* *}$ \\
3-15mins & $\mathrm{P}<0.002^{* *}$ & $\mathrm{P}<0.001^{* *}$ & $\mathrm{P}<0.01^{*}$ \\
4-20mins & $\mathrm{P}<0.006^{* *}$ & $\mathrm{P}<0.01^{*}$ & $\mathrm{P}<0.01^{*}$ \\
5-25mins & $\mathrm{P}<0.000^{* * *}$ & $\mathrm{P}<0.000^{* * *}$ & $\mathrm{P}<0.01^{*}$ \\
6-30mins & $\mathrm{P}<0.000^{* * *}$ & $\mathrm{P}=0.583 \mathrm{n} . \mathrm{s}$ & $\mathrm{P}<0.001^{* *}$ \\
7-35mins & $\mathrm{P}<0.002^{* *}$ & $\mathrm{P}=0.526 \mathrm{n} . \mathrm{s}$ & $\mathrm{P}<0.001^{* *}$ \\
8-40mins & $\mathrm{P}<0.000^{* * *}$ & $\mathrm{P}=0.261 \mathrm{n} . \mathrm{s}$ & $\mathrm{P}<0.01^{*}$ \\
9-45mins & $\mathrm{P}<0.000^{* * *}$ & $\mathrm{P}=0.336 \mathrm{n} . \mathrm{s}$ & $\mathrm{P}<0.01^{*}$ \\
10-50mins & $\mathrm{P}<0.000^{* * *}$ & $\mathrm{P}=0.475 \mathrm{n} . \mathrm{s}$ & $\mathrm{P}<0.02^{*}$ \\
11-55mins & $\mathrm{P}=0.584 \mathrm{n} . \mathrm{s}$ & $\mathrm{P}<0.01^{*}$ \\
12-60mins & $\mathrm{P}<0.000^{* * *}$ & $\mathrm{P}=0.586 \mathrm{n} . \mathrm{s}$ & $\mathrm{P}<0.02^{*}$ \\
\hline
\end{tabular}


Table (9) shows heart rate in study groups. "PACU". There were highly significance difference $(\mathrm{P}<0.000)$ between groups at different times.

When comparing between group $\mathrm{N} \&$ groupM. There were non significance difference $(\mathrm{P}>0.05)$ at different times.

When comparing between group N\& groupD. There were highly significance difference $(\mathrm{P}<0.000)$ at 5-
$30 \mathrm{mns}$., also there were moderate significance difference $(\mathrm{P}<0.001)$ at times $35-55 \mathrm{mns}$. As regard there were significance difference $(\mathrm{P}<0.05)$ at time 20 , there were non significance difference $(\mathrm{P}>0.05)$ at other times.

When comparing group $M \&$ groupD there were moderate significance difference $(\mathrm{P}<0.001)$ at times 60 mins. Also, there were highly significance difference $(\mathrm{P}<0.000)$ at other times.

Table (9): Heart Rate in beat/min in study groups. "PACU"

\begin{tabular}{|l|c|c|c|c|}
\hline \multicolumn{1}{|c|}{ Item } & $\mathbf{N}$ & $\mathbf{M}$ & $\mathbf{D}$ & P-value \\
\hline 1-5mins & $92.10 \pm 9.33$ & $79.53 \pm 9.96$ & $73.00 \pm 8.60$ & $\mathrm{P}<0.000^{* * *}$ \\
2-10mins & $87.50 \pm 8.08$ & $77.53 \pm 8.40$ & $74.13 \pm 6.50$ & $\mathrm{P}<0.000^{* * *}$ \\
3-15mins & $86.60 \pm 8.07 *$ & $77.06 \pm 8.07$ & $74.26 \pm 6.04$ & $\mathrm{P}<0.000^{* * *}$ \\
4-20mins & $86.20 \pm 8.57^{*}$ & $76.67 \pm 8.74$ & $73.20 \pm 5.01$ & $\mathrm{P}<0.000^{* * *}$ \\
5-25mins & $86.60 \pm 6.68^{*}$ & $79.00 \pm 8.63$ & $73.00 \pm 5.25$ & $\mathrm{P}<0.000^{* * *}$ \\
6-30mins & $86.90 \pm 8.11^{*}$ & $78.20 \pm 7.86$ & $74.63 \pm 6.36$ & $\mathrm{P}<0.000^{* * *}$ \\
7-35mins & $86.10 \pm 8.47^{*}$ & $78.00 \pm 8.65$ & $75.76 \pm 6.27$ & $\mathrm{P}<0.000^{* * *}$ \\
8-40mins & $86.20 \pm 8.37 *$ & $79.06 \pm 8.69$ & $76.76 \pm 6.71$ & $\mathrm{P}<0.000^{* * *}$ \\
9-45mins & $86.00 \pm 8.42^{*}$ & $79.00 \pm 8.36$ & $77.33 \pm 6.33^{*}$ & $\mathrm{P}<0.000^{* * *}$ \\
10-50mins & $86.10 \pm 8.76^{*}$ & $78.73 \pm 8.34$ & $77.86 \pm 6.47^{*}$ & $\mathrm{P}<0.000^{* * *}$ \\
11-55mins & $86.50 \pm 8.53^{*}$ & $78.93 \pm 8.39$ & $78.16 \pm 6.52^{*}$ & $\mathrm{P}<0.000^{* * *}$ \\
12-60mins & $87.20 \pm 8.27 *$ & $80.06 \pm 8.28$ & $78.06 \pm 7.43^{*}$ & $\mathrm{P}<0.000^{* * *}$ \\
\hline
\end{tabular}

Intragroup:*significant

**moderetly significant

***highly significant

\begin{tabular}{|c|c|c|c|}
\hline Item & P1\&P2 & P1\&P3 & P2\&P3 \\
\hline $1-5 \mathrm{mins}$ & $\mathrm{P}=0.155 \mathrm{n} . \mathrm{s}$ & $\mathrm{P}<0.000 * * *$ & $\mathrm{P}<0.000 * * *$ \\
\hline $2-10 \mathrm{mins}$ & $\mathrm{P}=0.758 \mathrm{n} . \mathrm{s}$ & $\mathrm{P}<0.000 * * *$ & $\mathrm{P}<0.000 * * *$ \\
\hline 3-15mins & $\mathrm{P}=0.517 \mathrm{n} . \mathrm{s}$ & $\mathrm{P}<0.000 * * *$ & $\mathrm{P}<0.000 * * *$ \\
\hline 4-20mins & $\mathrm{P}=0.083 \mathrm{n} . \mathrm{s}$ & $\mathrm{P}<0.000 * * *$ & $\mathrm{P}<0.002 * *$ \\
\hline $5-25 \mathrm{mins}$ & $\mathrm{P}=1$ & $\mathrm{P}<0.000 * * *$ & $\mathrm{P}<0.000 * * *$ \\
\hline 6-30mins & $\mathrm{P}=0.40 \mathrm{n} . \mathrm{s}$ & $\mathrm{P}<0.000 * * *$ & $\mathrm{P}<0.000 * * *$ \\
\hline 7-35mins & $\mathrm{P}=0.905 \mathrm{n} . \mathrm{s}$ & $\mathrm{P}<0.001 * *$ & $\mathrm{P}<0.000 * * *$ \\
\hline $8-40 \mathrm{mins}$ & $\mathrm{P}=0.256 \mathrm{n} . \mathrm{s}$ & $\mathrm{P}<0.002 * *$ & $\mathrm{P}<0.000 * * *$ \\
\hline $9-45 \mathrm{mins}$ & $\mathrm{P}=0.729 \mathrm{n} . \mathrm{s}$ & $\mathrm{P}<0.002 * *$ & $\mathrm{P}<0.000 * * *$ \\
\hline 10-50mins & $\mathrm{P}=0.655 \mathrm{n} . \mathrm{s}$ & $\mathrm{P}<0.001 * *$ & $\mathrm{P}<0.000 * * *$ \\
\hline $11-55 \mathrm{mins}$ & $\mathrm{P}=0.694 \mathrm{n} . \mathrm{s}$ & $\mathrm{P}<0.001 * *$ & $\mathrm{P}<0.000 * * *$ \\
\hline $12-60 \mathrm{mins}$ & $\mathrm{P}=0.625 \mathrm{n} . \mathrm{s}$ & $\mathrm{P}<0.000 * * *$ & $\mathrm{P}<0.001 * *$ \\
\hline
\end{tabular}

Table (10) shows complication in study groups. There were highly significance difference $(\mathrm{P}<0.000)$ between different groups with blood transfusion Bradycardia, Atropine and Esmolol. As regard there were moderate significance difference $(\mathrm{P}<0.001)$ with blood loss, Tachycardia.

Table (10): Complication in study groups.

\begin{tabular}{|l|c|c|c|c|}
\hline \multicolumn{1}{|c|}{ Item } & $\mathbf{N}$ & $\mathbf{M}$ & $\mathbf{D}$ & P-value \\
\hline Blood loss & $478.00 \pm 135.02$ & $386 \pm 200.06$ & $363.33 \pm 180.48$ & $\mathrm{P}<0.001^{* *}$ \\
Blood transfusion (ml) & & & & \\
Tachycardia & $700.00 \pm 258.19$ & $500.00 \pm 0.00$ & $377.50 \pm 44.59$ & $\mathrm{P}<0.000^{* * *}$ \\
Bradycardia & $18(60.0 \%)$ & $9(30.0 \%)$ & $4(13.3 \%)$ & $\mathrm{P}<0.001^{* *}$ \\
Atropine & 0.0 & $10(33.3 \%)$ & $24(80.0 \%)$ & $\mathrm{P}<0.000^{* * *}$ \\
Esmolol & 0.0 & $9(30.0 \%)$ & $21(70.0 \%)$ & $\mathrm{P}<0.000^{* * *}$ \\
& $16(53.3 \%)$ & $8(26.6 \%)$ & $3(10.0 \%)$ & $\mathrm{P}<0.000^{* * *}$ \\
\hline
\end{tabular}




\section{DISCUSSION}

The present study was designed to compare the effects of nitroglycerine, magnesium sulfate and dexmedetomidine as hypotensive agent in lumbar spine surgery.

As regarding systolic blood pressure, there was significant statistical difference at time of induction, at start of hyptensive agent, at 15 minute and at 30 minute intraoperative with blood pressure lower in dexmedetomidine group followed by magnesium sulfate group followed by nitroglycerine group.

As regarding diastolic blood pressure there was significant statistical difference at 30 minutes with blood pressure lower in dexmedetomidine followed by magnesium sulfate group and then nitroglycerine group.

As regarding heart rate there was significant statistical difference at start of hypotensive agent, at 15 minutes and after extubation with heart rate lower in dexmedetomidine followed by magnesium sulfate group and then nitroglycerine group.

However, in early postoperative period there was statistically significant difference in systolic blood pressure in all values with blood pressure lower in dexmedetomidine followed by magnesium sulfate group and then nitroglycerine group.

There was significant stastical difference in diastolic blood pressure in all values with blood pressure lower in dexmedetomidine group followed by magnesium sulfate and then nitroglycerine group.

There was significant statistical difference in heart rate in all values with heart rate lower in dexmedetomidine group followed by magnesium sulfate group and then nitroglycerin group.

These results of haemodynamics confirm that the haemodynamic profile of dexmedetmidine was steadier which can be attributed to the known sympatholytic effect of $\alpha_{2}$ agonist.

This study agrees with Elsharnouby \& Elsharnouby ${ }^{(12)}$ who studied patients scheduled for endoscopic surgery and found statistically significant lower heart rate and mean blood pressure in magnesium sulfate group with greater quality of vision of the surgical field and shorter operative time compared with control group.

Jamaliya et al. ${ }^{(13)}$ found that continuous infusion of dexmedetomidine is effective in minimizing blood loss and maintaining superior haemodynamics as comparied with nitroglycerine in posterior fixation spine surgery.

Nasreen et al. (14) in study assessing the hypotensive effect of dexmedetomidine administered $\mathrm{s}$ a $0.4 \mu \mathrm{g} / \mathrm{kg} / \mathrm{h}$ IV infusion following a $1 \mu \mathrm{g} / \mathrm{kg}$ IV bolus dose in middle ear surgery, it has been reported that surgeon satisfaction was increased and inhalation agent necessity to decrease the mean arterial pressure up to $30 \%$ was decreased in dexmedetomidine administered patient group.

Secondary decrease in the heart rate and blood pressure caused by dexmedetomidine is considered to be responsible for this situation.

In study conducted by Vali et al. ${ }^{(15)}$ comparing dexmedetomidine with nitroglycerine in patients undergoing posterior fixation surgery after traumatic spine fractures dexmedetomidine had better control over vital parameters e.g heart rate, mean arterial pressure, systolic blood pressure and diastolic blood pressure than nitroglycerine.

In study conducted by Rokhtabnak et al. ${ }^{(16)}$ compring dexmedetomidine with magnesium sulfate, blood pressure control was easier in the dexmedetomidine group that's number of patients that required nitroglyerine or analgesic rescue administration was lower in dexmedetomidine group.

As regard duration of surgery there was stastically significant difference with duration of surgery shorter in dexmedetomidine group followed by magnesium sulfate group then nitroglycerine group.

These results suggest that dexmedetomidine is the best regard visual field and duration of surgery.

With agreement to these results, study conducted by Vali et al. (15) to compare dexmedetomidine and nitroglycerine in posterior fixation surgery following traumatic spine surgery showed that surgeries in nitroglycerine group lasted for longer duration than dexmedetomidine group with difference being stastically significant.

As regarding blood loss there wes stastically significant difference with blood loss lower in dexmedetomidine group followed by magnesium sulfate group and then nitroglycerine group.

As regarding blood transfusion there was stastically significant difference with blood transfusion there was stastically significant difference with blood transfusion lower in dexmedetomidine group followed by magnesium sulfate group then nitroglycerine group.

As regard fromme score there was stastically significant difference with best result in dexmedetomidine group followed by magnesium sulfate group and then nitroglycerine group.

These results confirm that dexmedetomidine give good visual field with less blood loss and less blood transfusion requirements.

With agreement to this study, study conducted

by Bayram et al. ${ }^{(17)}$ comparing nitroglycerine with magnesium sulfate and dexmedetomidine during functional endoscopic sinus surgery showed that bleeding score was significantly decrease in group D.

Another study conducted by Vali et al. ${ }^{(15)}$ comparing dexmedetomidine with nitroglycerine in posterior fixation surgery following traumatic spine 
surgery stated that the mean blood loss was significantly lesser in dexmedetomidine group than nitroglycerine group. The requirement of blood transfusion was significantly more in nitroglycerine group than dexmedetomidine group.

As regard tachycardia there was stastically significant difference with the highest in nitroglycerine group followed by magnesium sulfate group and then dexmedetomidine group.

As regard hypotension there was stastically significant difference with the highest in dexmedetomidine group followed by magnesium sulfate group and then nitroglycerine group.

As regard bradycardia there was stastically significant difference with the highest in dexmedetomidine group followed by magnesium sulfate group and then nitroglycerine group.

These results demonstrate that hypotension and bradycardia are adverse effects of dexmedetomidine, tachycardia is adverse effect of nitroglycerine.

With agreement to this study, a study conducted by Vali et al. (15) comparing dexmedetomidine with nitroglycerine in posterior fixation following traumatic spine injury showed that patients in nitroglycerine group had more episodes of tachycardia compared to dexmedetomidine group during the period of observation, also in nitroglycerine group none of the patients had episode of hypotension or bradycardia where as in dexmedetomidine group 1 patient had hypotension and 2 patients had bradycardia, but which were not stastically significant.

In the study by Bayram $\boldsymbol{e t}$ al. ${ }^{\left({ }^{(17)}\right.}$ comparing dexmedetomidine and magnesium sulfate in functional endoscopic sinus surgery bradycardia occurred in 4 patients in dexmedetomidine group versus 1 patient in magnesium sulfate group.

In another study conducted by Ghodraty $\boldsymbol{e t} \boldsymbol{a l}$. (18) comparing magnesium sulfate and remifentanil there were 4 episodes of hypotension in magnesium sulfate group and 3 episodes in remifentanil group requiring pharmacological intervension (ephedrine boluses), a total of 3 patients in magnesium sulfate group and 1 patient in remfentanil group received atropine due to bradycardia.

As regard time required to restore blood pressure to baseline after discontinuation of hypotensive drug there was stastically significant difference with the time longer in dexmedetomidine group followed by magnasium sulfate group and then nitroglycerine group.

These results show that dexmedetomidine acts by selectively binding to $\alpha_{2}$ receptors with great affinity while nitroglycerine prodoce its hypotensive action by liberating nitric oxide which has half life of 0.1 seconds.
Sedative effect of magnasium sulfate were attributed to its ability to inhibit NMDA receptors in non compatative way.

With agreement to this study a study conducted by Khalifa \& Awad ${ }^{(19)}$ comparing dexmedetomidine, magnasium sulfate and nitroglycerine during functional endoscopic sinus surgery showed that significant difference between the groups was present in analysis of the time needed to restore the basal values of mean arterial pressure with longer time in dexmedetasiumomidine group.

Another study conducted by Jamaliya et al. ${ }^{(13)}$ comparing dexmedetomidine and nitroglycerine during posterior fixation surgery following traumatic spine injury showed that time to reversibility of the hypotensive state was lesser in the nitroglycerine group when compared to dexmedetomidine group.

As regard time to first analgesic rescue there was stastically significant difference with time longer in dexmedetomidine group followed by magnesium sulfate group and then nitroglycerine group.

These results demonstrate the analgesic properities of both drugs.

With agreement to this study a study conducted by Khalifa \& Awad ${ }^{(19)}$ comparing dexmedetomidine, magnesium sulfate and nitroglycerine during functional endoscopic sinus surgery showed that longer time to require postoperative analgesia in the dexmedetomidine group and magnesium sulfate group compared with nitroglycerine group.

Another study conducted by Somayaji and Raveendra ${ }^{(20)}$, studying effect of dexmedetomidine on blood loss and quality of surgical field in functional endoscopic sinus surgery showed that postoperatively, patients receiving dexmedetomidine didn't report pain for longer period where`s time to first analgesic request for the patients in the control group was much shorter. This was found to be stastically significant.

\section{CONCLUSION}

We concluded that nitroglycerine, magnesium sulfate and dexmedetomidine could induce hypotension, but dexmedetomidine showed more favorable hemodynamic profile as regard blood pressure and heart rate.

Dexmedetomidine also showed shorter duration of surgery with less blood loss, less blood transfusion and favorable frommer score with more surgeon satisfaction.

Dexmedetomidine showed longest time to $1^{\text {st }}$ analgesic rescue in PACU comparing with magnesium sulfate and nitroglycerine.

So, it is advised to use dexmedetomidine to induce hypotension than magnesium sulfate and nitroglycerine.

However, Dexmedetomidine should be used with caution as it caused hypotension and bradycardia, 
it had sedative effect with low aldert score comparing with nitroglycerine and magnesium sulfate.

\section{REFERENCES}

1. Wylie CD (2003): A Practice of Anaesthesia, in A Practice Of Anaesthesia, P.R.K. Thomas E J Healy, Editor. Arnold. https://www.abebooks.com/servlet/BookDetailsPL?bi=30 182152999\&searchurl=isbn\%3D9780340731307\%26sort by\%3D17\&cm_sp=snippet-_-srp1-_-image1

2. Nilesh J, Patel B (1985): Induced moderate hypotensive anaesthesia for spinal fusion and Harrington rod instrumentation. The Journal of Bone and Joint Surgery, 67(9): 1384-1387.

3. Ward CF, Alfery David D, Saidman LJ (2006): Deliberate hypotension in head and neck surgery. https://onlinelibrary.wiley.com/doi/abs/10.1002/hed.2890 020304

4. Degaute CS (2007): Controlled hypotension: a guide to drug choice. Drugs, 67: 1053-1076.

5. Guggiari M, Dagreou F, Lienhart A et al. (1985): Use of nitroglycerine to produce controlled decreases in mean arterial pressure to less than $50 \mathrm{~mm} \mathrm{Hg}$. Br J Anaesth., 57(2): 142-147.

6. Testa LD, Tobias JD (1995): Pharmacologic drugs for controlled hypotension. J Clin Anesth., 7: 326-337.

7. Koinig H, Wallner T, Marhofer $P$ et al. (1998): Magnesium sulfate reduces intra- and postoperative analgesic requirements. Anesth Analg., 87:206-210.

8. Granry JC, Rod B, Monrigal JP et al. (2003): The analgesic efficacy of an injectable prodrug of acetaminophen in children after orthopaedic surgery. Pediatric Anesthesia, 7:445-449.

9. Virtanen N, Savola JM, Saano V et al. (1988): Characterization of the selectivity, specificity and potency of medetomidine as an alpha 2-adrenoceptor agonist. Eur J Pharmacol., 150: 9-14.

10. Bloor BC, Ward DS, Belleville JP et al. (1992): Effect of intravenous dexmedetomidine in humans. II. Hemodynamic changes. Anesthesiology, 77: 1134-1142.

11.Lakhlani PP, MacMillan LB, Guo TZ et al. (1997): Substitution of a mutant alpha $2 \alpha$-adrenergic receptor subtype in sedative, analgesic, and anaesthetic sparing responses in vivo. Proc Natl Acad Sci USA., 94: 99509955.

12.Elsharnouby NM, Elsharnouby MM (2006): Magnesium sulphate as a technique of hypotensive anaesthesia. BJA: British Journal of Anaesthesia, 96(6): 727-731.

13. Jamaliya RH, Chinnachamy R, Maliwad J et al. (2014): The efficacy and hemodynamic response to Dexmedetomidine as a hypotensive agent in posterior fixation surgery following traumatic spine injury. Journal of Anaesthesiology, Clinical Pharmacology, 30(2):.203-7.

14. Nasreen F, Bano S, Khan RM et al. (2009): Dexmedetomidine used to provide hypotensive anesthesia during middle ear surgery. Indian Journal of Otolaryngology and Head \& Neck Surgery, 61(3): 205207.

15. Vali LF, Gedam NT, Vali AH (2017): Anesthesia management in a giant congenital undifferentiated orbital teratoma: A challenging, rare entity. Indian Anaesthetists Forum, 18: 28-30.

16. Rokhtabnak F, Motlagh SD, Ghodraty M et al. (2017): Controlled Hypotension During Rhinoplasty: A Comparison of Dexmedetomidine with Magnesium Sulfate. Anesthesiology and Pain Medicine, 7(6): e64032.

17. Bayram A, Ülgey A, Günes I, Ketenci I et al. (2015): Comparison between magnesium sulfate and dexmedetomidine in controlled hypotension during functional endoscopic sinus surgery. Revista Brasileira de Anestesiologia, 65(1): 61-67.

18. Ghodraty MR, Homaee MM, Farazmehr $\mathrm{K}$ et al. (2014): Comparative induction of controlled circulation by magnesium and remifentanil in spine surgery. World Journal of Orthopedics, 5(1):51-56.

19. Khalifa OS, Awad OG (2015): A comparative study of dexmedetomidine, magnesium sulphate, or glyceryl trinitrate in deliberate hypotension during functional endoscopic sinus surgery. Ain-Shams Journal of Anaesthesiology, 8(3): 320-25.

20.Somayaji A, Raveendra US (2016): Effect of dexmedetomidine on blood loss and quality of surgical field in functional endoscopic sinus surgery: A double blinded prospective controlled study. Karnataka Anaesthesia Journal, 2(3): 90-98. 